\title{
Automatic Tabulation of Generically Immersed Intervals and Their Associated Links
}

\author{
Andrew GIBSON* and William GIBSON ${ }^{1}$ \\ ${ }^{1}$ Graduate School of Mathematical Sciences, University of Tokyo, 3-8-1 Komaba, Meguro-ku, Tokyo 153-8914, Japan \\ E-mail: gibson@ms.u-tokyo.ac.jp
}

Received July 1, 2002; final version accepted January 31, 2003

\begin{abstract}
We introduce an algorithm for generating all generically immersed intervals in the unit disk with a given number of double points $N$ and modulo mirror images. These interval immersions, known as free divides, are associated with knots via a particular map into the 3 -sphere, and the gordian unknotting number of a link of a free divide is equal the number of double points of the immersion. So the algorithm provides access to knots for which the gordian number is explicitly known. A computer implementation of our algorithm is described, and we report the results for the total numbers of free divides for $N \leq 8$.
\end{abstract}

KEYWORDS: free divide, knot theory, gordian number, distributed computing

\section{Introduction}

In this paper we describe an algorithm for generating all possible generic immersions of an interval in the unit disk with a specified number of double points. The motivation for doing this arises from the study of objects called divides and free divides which we briefly describe. Divides were originally used in the study of real morsifications of complex plane curve singularities (see the works of A'Campo [1,2] and Gusein-Zade [8-10]), and they are defined as follows.

Definition 1.1. A divide is a generic relative immersion of unit intervals and circles in the unit disk $D$. Here relative describes the condition that all the end-points of each interval (and only those end-points) are mapped to the boundary of $D$, and by generic we mean that every multiple point is a transversal double point, and that the end-points of intervals are transversal to the disk boundary.

Each divide is associated with a knot or link in the 3-sphere $S^{3}$ via a particular map, and under certain conditions these links enjoy nice properties. Two of these properties (proved in [3]) are as follows:

(1) The link of a connected divide is always fibered;

(2) If the divide contains only immersed intervals, then the gordian number (a particular type of unknotting number) is given by the number of double points of the immersion.

In an attempt to extend these two properties to a larger class of links, the second author and Ishikawa [7] defined and studied free divides. A free divide is defined in exactly the same way as a divide, except we no longer insist that the immersion be relative. It was shown in that paper that the gordian number result does extend to all free divide comprised of interval immersions. However, in tabulating all free divides up to 3 double-points (and a sub-class of free divides with 4 double-points), it was found that the fibrability result does not extend in general. Nevertheless, since the gordian number is difficult to determine in general, the motivation for tabulating free divides for a given number of double-points (i.e. a given gordian number) is clear.

In order to easily describe the configuration of a given free divide we consider the following construction.

Definition 1.2. Let $f: I \hookrightarrow D$ be a generic immersion of the oriented unit interval $I=[0,1]$ into the unit disk $D$ given by $D:=\left\{\mathbf{x} \in \mathbb{R}^{2}|| \mathbf{x} \mid \leq 1\right\}$. We will call the image $f(I) \in D$ a path, and define $S:=f(0)$ and $E:=f(1)$ to be the start and end of the path respectively.

By definition, a path in the unit disk is a free divide equipped with an orientation. Thus, if we let $\mathfrak{F}$ represent the set of isotopy classes of free divides, and let $\mathfrak{P}$ represent the isotopy classes of paths in the plane, then we have a welldefined map $\mathfrak{f}: \mathfrak{P} \rightarrow \mathfrak{F}$ which simply strips the orientation data from paths.

We will now present a brief summary of the relevant properties of free divides. The reader is referred to [7] for the proofs.

Links of free divides are defined via doublings. Let $F$ be a free divide and $k \in \mathbb{Z}_{0}^{+}$a non-negative integer. A choice of doubling $F_{k}$ of $F$ is constructed by replacing each arc of $F$ with a pair of parallel arcs, capping the two ends with semicircles, and introducing $k$ 'twists' at arbitrary points in the new diagram. An orientation is then assigned so that the 


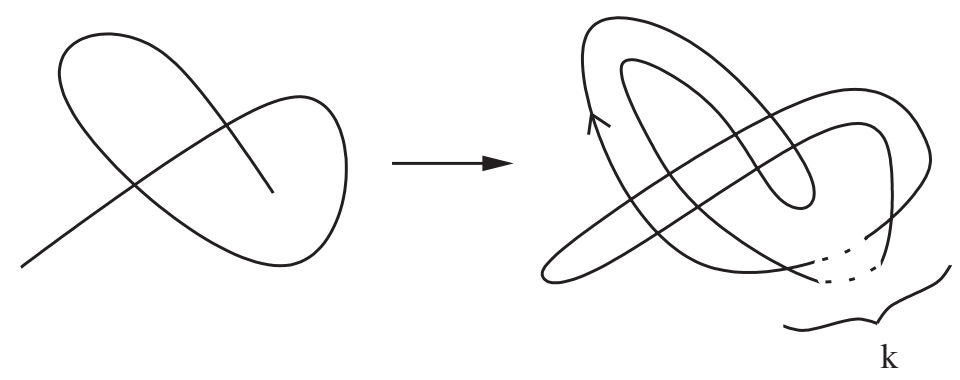

Fig. 1. Example of doublings of a free divide.

doubling can be interpreted as an oriented divide. Figure 1 describes this doubling process pictorially.

A link $L\left(F_{k}\right)$ of the free divide $F$ is then defined to be the link of a choice of doubling, i.e.

$$
L\left(F_{k}\right)=\left\{\left.(\mathbf{x}, \mathbf{u}) \in \mathbb{R}^{4}\left|\mathbf{x} \in F_{k} \subset D, \mathbf{u} \in \vec{T}_{\mathbf{x}} F_{k},\right| \mathbf{x}\right|^{2}+|\mathbf{u}|^{2}=1\right\},
$$

where $\vec{T}_{\mathbf{x}} F_{k}$ is the set of speed vectors of $F_{k}$ at $\mathbf{x}$ which coincide with the orientation on $F_{k}$.

By considering ambient isotopy equivalence of links of free divides it can be shown that the integer $k$ need only be considered modulo two. Moreover, if a free divide $F$ has at least one end-point that can be isotoped to the boundary of the unit disk, then the links of either doubling of $F$ are ambient isotopic. This is not true in general for free divides whose end-points cannot be isotoped to the boundary of the disk.

Another important consideration is that of mirror images of free divides: If the free divide $F^{*}$ is the mirror image of $F$ then $L\left(F_{k}^{*}\right)$ is ambient isotopic to $L\left(F_{k}\right)$. This is shown by observing that one link is obtained from the other by two reflections (and hence a rotation) in $S^{3}$. Thus if we are solely interested in ambient isotopy of links of free divides then we can justifiably ignore mirror images of free divides. This 'isotopy symmetry' clearly lifts to paths via $\mathfrak{f}$, i.e. if $P^{*}$ is the mirror image of any path $P$, then $L\left(\mathfrak{f}\left(P_{k}^{*}\right)\right)$ is ambient isotopic to $L\left(\mathfrak{f}\left(P_{k}\right)\right)$ for either choice of $k \in \mathbb{Z} / 2 \mathbb{Z}$.

The algorithm we describe in the sequel automatically tabulates all possible free divides with a given number of double points, modulo mirror images. The method involves preparing a list of paths with $N$ double points in a systematic way, and then eliminating symmetries arising from orientation reversal and mirror images.

As a by-product of free divide tabulation, the computer implementation of the algorithm described in this paper provides us with the total number of free divides with a given number of double points. The totals for divides and free divides up to unknotting number 8 are displayed in Table 1. In this table, the subtotals for prime divides and free divides are also shown. A free divide $P$ in the unit disk $D$ is prime if there does not exist a proper embedding $I$ of the unit interval in the disk such that

(1) $I$ intersects $P$ exactly once,

(2) both components of $D \backslash I$ contain at least one double point of $P$.

If such an interval does exist then we say that $P$ is a compound free divide, and write $P=P_{1} \sharp P_{2}$, where $P_{1}$ and $P_{2}$ are the closures of the two components of $P \backslash I$. It can then be shown that the link of $P$ is the connected sum of the links of $P_{1}$ and $P_{2}$, i.e.

$$
L\left(P_{1} \sharp P_{2}\right)=L\left(P_{1}\right) \sharp L\left(P_{2}\right) .
$$

An example of a compound free divide is shown in Fig. 2 together with it's doubling and link. The method for obtaining the link from the doubling is described in detail in $[5,6]$. Note that this link is clearly a connected sum (in fact, the connected sum of two right-handed trefoils).

It follows that if a free divide knot is prime then every free divide representation of it is also prime. However, the
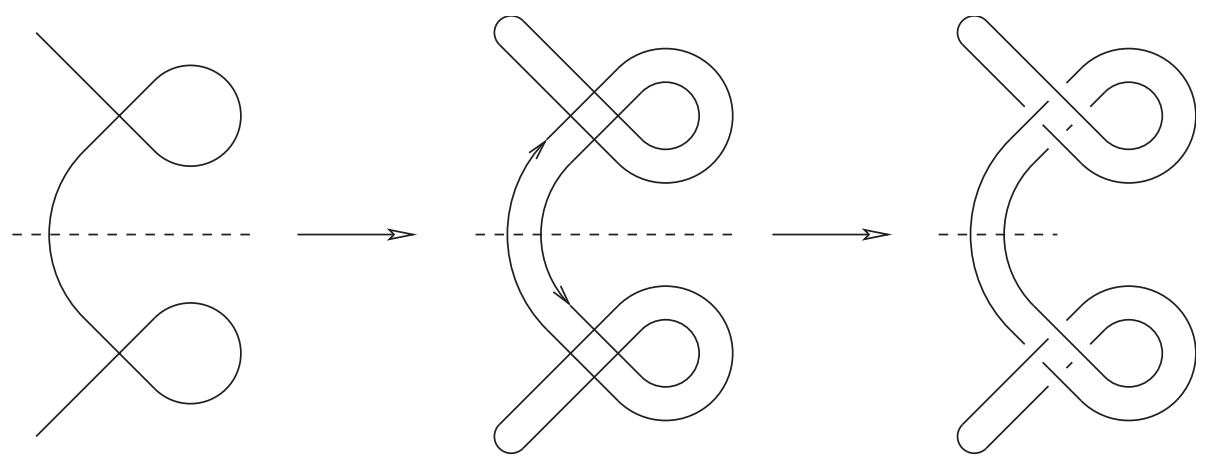

Fig. 2. Example of a compound divide, its doubling and its link. 
converse of this statement is not true in general. For example, the free divide shown in Fig. 3 (free divide 26 from the tables in [7]) is prime, but the link of its even doubling is also the connected sum of two right-handed trefoils.

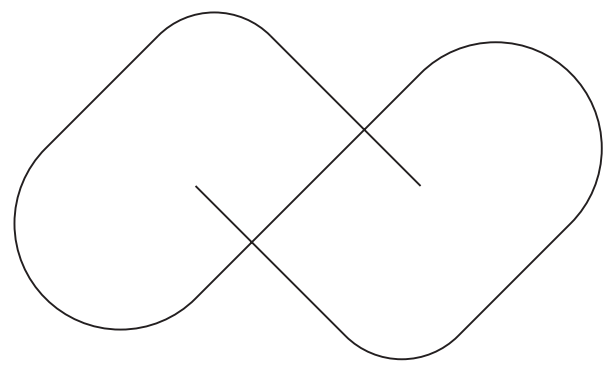

Fig. 3. The prime free divide 26 . The link of its even doubling is not a prime knot.

Table 1. The number of divides and free divides up to 8 double points (modulo mirror images). Values written in italic numerals were calculated independently in [7].

\begin{tabular}{|c|r|r|r|r|}
\hline \multirow{2}{*}{$\sharp$ double points } & \multicolumn{2}{|c|}{$\sharp$ divides } & \multicolumn{2}{|c|}{$\sharp$ free divides } \\
\cline { 2 - 5 } & prime & total & prime & total \\
\hline 1 & 1 & 1 & 2 & 2 \\
2 & 2 & 4 & 10 & 12 \\
3 & 8 & 15 & 70 & 79 \\
4 & 38 & 82 & 604 & 678 \\
5 & 223 & 496 & 5687 & 6287 \\
6 & 1534 & 3522 & 56824 & 62232 \\
7 & 11711 & 26950 & 583174 & 633172 \\
8 & 95971 & 219593 & 6090648 & 6566426 \\
\hline
\end{tabular}

Remark 1. The software implementation of our algorithm is freely available. Anyone interested in obtaining a copy is invited to contact the corresponding author.

\section{Acknowledgements}

Both authors thank the referees for various suggestions incorporated into the final version of this paper. In addition, the second author would like to thank Toshitake Kohno for his support in this project.

This work was supported by a Scholarship from the Ministry of Education, Culture, Sports, Science and Technology of Japan.

\section{Path Descriptions}

Let $\mathfrak{G}$ be the set of all generic immersions of the oriented unit interval in the plane. For any two paths $P_{1}$ and $P_{2}$, define $\sim$ to be the equivalence relation such that $P_{1} \sim P_{2}$ if and only if $P_{1}$ is isotopic to $P_{2}$ (i.e. equivalent in the theory of plane curves where triple crossings and self-tangencies are forbidden). Then $\mathfrak{P}:=\mathfrak{G} / \sim$ is a set of descriptions of all possible isotopy classes of paths.

Let $P \subset D$ be any path, and let $[P] \in \mathfrak{P}$ be the isotopy class of paths containing $P$.

Definition 2.1. A path description of a path $P$ is an alphanumeric text string which encodes $[P]$, i.e. a sequence of text characters that (after appropriate decoding) gives a complete description of the isotopy class of $P$. A path description method $\mathfrak{M}$ is an injective map

$$
\mathfrak{M}: \mathfrak{P} \rightarrow \text { alphanumeric text strings }\}
$$

defined by a finite set of rules that generates path descriptions for all paths.

By defining $\mathfrak{M}(P):=\mathfrak{M}([P])$ it follows that $\mathfrak{M}\left(P_{1}\right)=\mathfrak{M}\left(P_{2}\right)$ if and only if $P_{1} \sim P_{2}$.

\section{Path Symmetries}

For any path $P$, let $\mathfrak{f}(P)_{k}$ be a choice of doubling for $P$ stripped of its orientation. The link of this doubling is $L\left(\mathfrak{f}(P)_{k}\right) \in S^{3}$. 
Definition 3.1. The mirror $P^{*}$ of $P$ is simply the mirror image of $P$. The reversal $-P$ of $P$ is $P$ with the opposite orientation. The mirror reversal $-P^{*}$ is given by $-\left(P^{*}\right) \sim(-P)^{*}$.

Two fundamental properties of links of paths concern the natural link symmetries of paths:

- $L\left(\mathfrak{f}\left(P^{*}\right)_{k}\right) \simeq L\left(\mathfrak{f}(P)_{k}\right)$.

- $L\left(\mathfrak{f}(-P)_{k}\right) \simeq L\left(\mathfrak{f}(P)_{k}\right)$.

The first statement is true because (up to ambient isotopy) the link on the left hand side is obtained from the one on the right by performing two reflections in $S^{3}$. The second isotopy is trivial since $\mathfrak{f}(-P)=\mathfrak{f}(P)$ by definition.

Thus any given free divide has up to four possible path descriptions. In addition to these properties, we are interested in two particular path symmetries:

Definition 3.2. A path $P$ has mirror symmetry if $P \sim-P^{*}$ and reversal symmetry if $P \sim-P$.

Note that mirror symmetry also implies $-P \sim P^{*}$, that reversal symmetry also implies $P^{*} \sim-P^{*}$, and that these are the only two possible symmetries. Thus the number of distinct path descriptions of a free divide $F$ is one, two or four, depending on its symmetry class. Table 2 summarizes the possible cases.

Table 2. Summary of path symmetries.

\begin{tabular}{|l|r|r|}
\hline symmetry & example & $\#$ distinct path descriptions \\
\hline none & 4 (i.e. $P, P^{*},-P,-P^{*}$ ) \\
mirror & 2 (i.e. $\left.P \sim-P^{*},-P \sim P^{*}\right)$ \\
mirror, reversal & 2 & (i.e. $\left.P \sim-P, P^{*} \sim-P^{*}\right)$ \\
\hline
\end{tabular}

It is fairly easy to see that the only free divide with both mirror and reversal symmetries is the trivial divide, i.e. the embedded interval (a proof of this fact is given in Remark 3 in Sect. 5.2). Hence, for any path description method, all free divides with at least one double point have at least two distinct path descriptions. In order to tabulate all free divides with a given number of double points, we wish to have just one path description for each divide.

\section{The Algorithm}

We now describe an algorithm which generates all possible path descriptions, without duplication, for a given number of double points $N \geq 1$. The basic scheme of the algorithm is as follows.

- Pick a suitable path description $\mathfrak{M}$;

- generate $\operatorname{Im} \mathfrak{M}$ for paths with $N$ double points;

- set $F:=\emptyset$;

- for each path description $[P] \in \operatorname{Im} \mathfrak{M}$ :

(1) compute the list $Q([P])=\left\{[P],\left[P^{*}\right],[-P],\left[-P^{*}\right]\right\}$;

(2) sort $Q([P])$ alphanumerically;

(3) set $A([P])$ to be the first element in the sorted list $Q([P])$;

(4) if $[P]=A([P])$ then set $F:=F \cup\{[P]\}$;

(5) discard $[P]$.

Theorem 4.1. On completion of the algorithm, the set $F$ contains a single representative path description for each immersed interval with $N$ double points, modulo mirror images.

Proof 1. Every path in $\operatorname{Im} \mathfrak{M}$ is unique. Given a path $[P]$ we show that of the list $Q([P])$ only one member will be added to the list of distinct paths and the others will be eliminated.

If $[P],\left[P^{*}\right],[-P]$ and $\left[-P^{*}\right]$ are all distinct then $A([P])$ is equivalent to only one of the four. Since $A([P])=$ $A\left(\left[P^{*}\right]\right)=A([-P])=A\left(\left[-P^{*}\right]\right)$ it follows that only one of the four will pass the test and be kept. The three that don't equal $A([P])$ will be eliminated.

If $[P]=[-P]$ then $\left[P^{*}\right]=\left[-P^{*}\right]$ then we only run the test against $[P]$ and $\left[P^{*}\right]$ once each. As $A([P])=A\left(\left[P^{*}\right]\right)$, only one will pass the test and the other will be eliminated. Similarly, if $[P]=\left[-P^{*}\right]$ then $\left[P^{*}\right]=[-P]$ then only one will 
pass the test.

This covers all cases, since $N \geq 1$ implies $[P] \neq\left[P^{*}\right]$.

\section{Implementation of the Algorithm}

The implentation of the algorithm actually uses two path description methods. The first method $\mathfrak{M}_{1}$, which adopts an intuitively easy approach to path building, is used to generate the list of valid paths. The second method $\mathfrak{M}_{2}$ is introduced to simplify the problem of constructing path descriptions of reversals, and is used in the elimination phase of the algorithm. In this section we define $\mathfrak{M}_{1}$ and $\mathfrak{M}_{2}$, explain how valid paths are generated, and show how the symmetries behave in both settings. We also describe the conversion map $C: \mathfrak{M}_{1} \rightarrow \mathfrak{M}_{2}$ used to pass between the two methods.

\subsection{The $\mathfrak{M}_{1}$ path description method}

Recall that an immersed interval is a path in a surface with a start and an end. The crossing points and end points are referred to as nodes. The end nodes are labelled $S$ and $E$ (for 'start' and 'end'), and the crossing nodes are numbered, starting from 0 , in the order that they are encountered along the path as we travel from $S$ to $E$.

Each section of the path that joins two nodes is called an edge. Each node representing a crossing of the path has four tips. The tips of a node are labelled back, front, left and right: The back tip is the one by which the path first encounters the node, and the remaining tips are labelled in the natural way, as shown in Fig. 4.

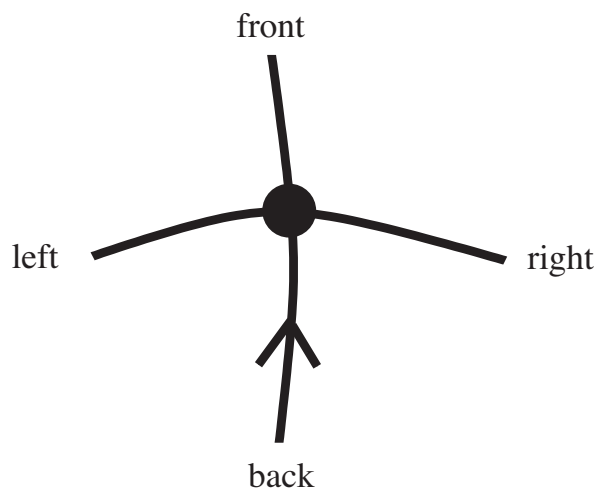

Fig. 4. Tip labels at a node.

A path can be built up from a recipe which describes how each edge can be appended to the edges already put together. At the simplest level an edge can do one of three things:

(1) It can loop back and join to a tip on the same node.

(2) It can join an unjoined node to the path.

(3) It can link to a tip on another node that's already in the path.

These three elements are examined more closely in turn and a notation is introduced to describe exactly how the edge is joined to the path.

Loops. A loop joins the front tip to either the left tip or the right tip of the same node. A left loop is a loop joining to the left tip. A right loop is similarly defined. If the loop isn't in a region completely enclosed by the path, the loop could also be anticlockwise or clockwise. For example a clockwise left loop completely encloses the path, whereas an anticlockwise one doesn't. For a loop in an internal region, the loop can only go in one direction so it isn't necessary to note which direction that is.

The notation for the loop instruction is

$$
P[L R]([A C]),
$$

where [ ] means choose one letter within the brackets and ( ) denotes an optional element. The $L$ or $R$ specifies whether the loop is a left or right one. The $A$ or $C$ specifies the direction (anticlockwise or clockwise).

Example 5.1. For a clockwise left loop the notation is $P L C$. For a clockwise right loop in an internal (enclosed) area the notation is $P R$ (as there couldn't be an anticlockwise right loop in an internal area).

Joins. A join connects the current tip to the back tip of a new node. The join instruction is designated by the letter $J$ and appended by the label of the new node, i.e. Jn. 
Example 5.2. For a join connecting the second crossing node, the notation is $J 1$.

Remark 2. In fact the letter $J$ by itself would be sufficient notation as the crossing nodes are numbered by the order in which they are joined. The node label is included in the notation for clarity.

Links. A link connects the current tip to a tip of an already joined node. As the node is already joined the only free tips will be the left or right tips. Therefore, just like a loop, a link can be a left link or a right link. Similarly, if the link is dividing the external region, it is important to know whether the link is going clockwise or anticlockwise around the path. For internal regions the direction is irrelevant.

The link instruction is designated by the letter $K$. The full notation is given by

$$
K n[L R]([A C]) \text {. }
$$

Here $n$ is the label of the node which is being linked. The $L$ or $R$ specifies whether the link is a left or right one. The $A$ or $C$ specifies the direction (anticlockwise or clockwise).

Example 5.3. For a link connecting to the second crossing node's left tip, going anticlockwise around the path the notation is $K 1 L A$. For an internal link connecting to the fourth crossing node's right tip, the notation is $K 3 R$.

A path can therefore be described as a sequence of instructions which describe how the edges are drawn as we go from the start node to the end node. The description of the path can then be written as a single line of text with hyphens separating each instruction.

Example 5.4. Path (a) in Fig. 5 can be described thus:

$$
J 0-J 1-K 0 L A-J 2-P L C-K 1 R-J E .
$$

(a)

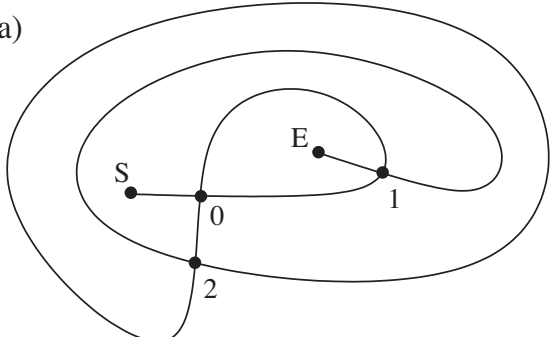

(c)

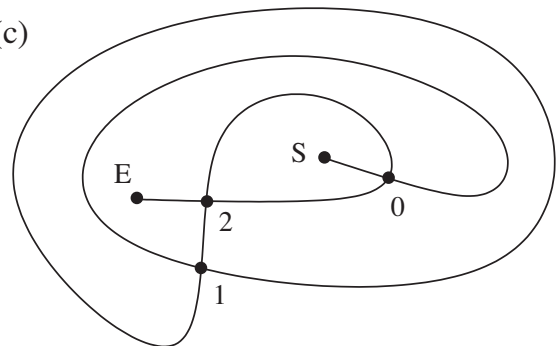

(b)

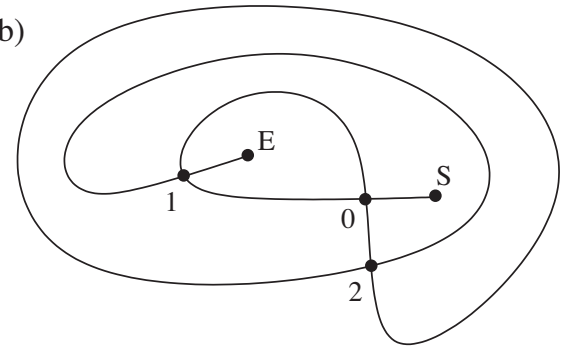

(d)

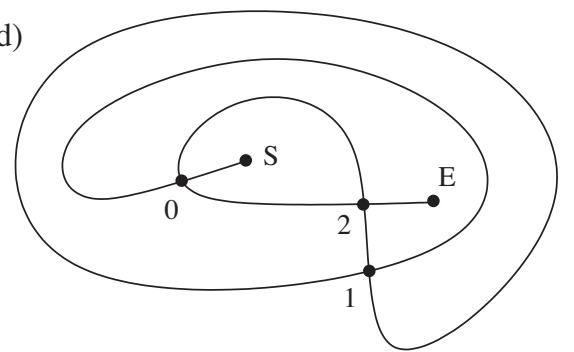

Fig. 5. Example of a path (a) with three crossings, together with its mirror (b), reversal (c), and mirror reversal (d).

As every path description will begin with $J 0$ and end with $J E$, these are omitted for conciseness to give:

$$
J 1-K 0 L A-J 2-P L C-K 1 R \text {. }
$$

The rules described above give a well-defined path description method $\mathfrak{M}_{1}$. Thus for any immersed interval $P$ we can generate a description $\mathfrak{M}_{1}(P)$ and, once we have a valid description, we can draw the immersed interval it describes.

\subsection{Mirrors and reversals via $\mathfrak{M}_{1}$}

The $\mathfrak{M}_{1}$ path description of a mirror image can be obtained from the original path description by making the following simple substitutions: 


$$
\begin{aligned}
& L \rightarrow R \\
& R \rightarrow L \\
& A \rightarrow C \\
& C \rightarrow A .
\end{aligned}
$$

Remark 3. Note that if there is at least one double point, the mirror of a path always has a different description to the original path. This is because the path always contains at least one $L$ or $R$. This provides a proof of the fact (mentioned at the end of Sect. 3) that the trivial path is the only path with both mirror and reversal symmetries.

As mentioned previously, the computation of the corresponding description of the reversal from the initial path description is more difficult, and we address this problem in Sect. 5.4.

Example 5.5. Displayed in Fig. 5 are four paths which are obtained from the same free divide. Paths (b), (c) and (d) are the mirror, reversal and mirror reversal of the initial path (a) respectively. The corresponding $\mathfrak{M}_{1}$ path descriptions are summarized in Table 3.

Table 3. The four path descriptions for the paths shown in Fig. 5.

\begin{tabular}{|c|c|c|}
\hline path & Fig. 5 & $\mathfrak{M}_{1}$ path description \\
\hline initial & (a) & $J 1-K 0 L A-J 2-P L C-K 1 R$ \\
mirror & (b) & $J 1-K 0 R C-J 2-P R A-K 1 L$ \\
reversal & (c) & $J 1-P R A-J 2-K 0 L-K 2 R$ \\
mirrored reversal & (d) & $J 1-P L C-J 2-K 0 R-K 2 L$ \\
\hline
\end{tabular}

\subsection{Generating all the paths with $\boldsymbol{N}$ crossings}

For $N$ crossings there are $2 N-1$ edges in each $\mathfrak{M}_{1}$ path description, and for each edge there is a finite number of possible descriptions. So we can build a finite tree where each node represents an initial part of a path. The root of this tree represents the empty path description. Then, given a node with an $\mathfrak{M}_{1}$ path description of $P$ and distance from the root less than $2 N-1$, we can, for each instruction $d$ (taken from the finite set of all such descriptions), create a new child node with path description ' $\mathfrak{M}_{1}(P)-d$ '.

As it stands, many branches in this tree will be invalid. For example the branch adding a description $J 2$ to any node representing an initial path description not containing the instruction $J 1$ will be invalid. Similarly other branches can be discounted because they would generate paths that couldn't be drawn. The algorithm checks which paths are actually valid by keeping track of the regions in the diagram and knows which tips are in which region (and so knows which can legitimately be joined up).

The external region is labelled 0 , and subsequent regions are labelled $1,2,3, \ldots$ As the program traverses the tree of paths, it maintains a tip table which holds the state of all the tips. The tip table knows whether a tip is connected or not, and what the tip is connected to if it is connected. Importantly, it also knows the region the tip was in before it was connected.

The first node is put in the external region so all its tips are in region 0. Now consider the three instructions:

Loops: If the front tip is free then a loop is possible as the left and right tips must be in the same region. A PLA, PRC, $P L$ or $P R$ instruction creates a new region which doesn't contain any tips. A PLC or PRA instruction (producing an edge which loops round the entire path) creates a new region which contains all the unconnected tips that were in region 0 . The tip table is updated to reflect this.

Joins: All the free tips ( $L, F$ and $R$ ) of a new node joined to from an $F$ tip in region $n$ will also be in region $n$.

Links: Two unconnected tips can only be linked if they are from different nodes but are in the same region. The new edge divides the region in two. One part of this region is designated the new region and the tip table is updated to reflect this.

Because the tip table keeps track of all connections it is possible to trace round the inner edge of any region to discover which unconnected tips are contained in it. In the case of links it is obviously possible, before making the link, to start at the proposed start point of the link and travel clockwise or anticlockwise around the internal edge of the region until reaching the proposed end point of the link, searching for unconnected tips (to update their region number). 


\subsection{The $\mathfrak{M}_{2}$ path description method}

As mentioned earlier, the difficulty of constructing path descriptions of reversals is overcome by introducing a second path description method. $\mathfrak{M}_{2}$ is a method such that $\mathfrak{M}_{2}(P)$ is a list of all the edges of $P$ including sufficient information for gluing these edges together in a unique way.

First we define an ordering on the tips given by

$$
S<0 F<0 L<0 R<0 B<1 F<1 L<1 R<1 B<\cdots<E .
$$

A list of 'edge descriptions' for $P$ is then generated by searching through all the crossing node tips in the diagram in this standard order. The method is as follows.

For each tip $T$, define $H(T)$ to be the edge of the path to which $T$ is attached, and $T^{\prime}$ to be the other tip that $H(T)$ is attached to (i.e. so that $H(T)=H\left(T^{\prime}\right)$ with $T \neq T^{\prime}$ ). Then for each edge $H$ in the path $P$ we define Type $(H(T)) \in$ $\{a, c, i, x\}$ as follows. If $H$ is an internal edge of $P$ then Type $(H)=i$ (for internal). For outside edges of $P$ we do the following. Starting from an arbitrary outside node we traverse the outer edges of $P$ in a clockwise direction. If during this traversal we travel along $H$ in both directions then Type $(H)=x$ (for external). Otherwise we can only have travelled along $H$ once. If we travelled in the same direction as the orientation of $H$ then Type $(H)=c$ (for $c l o c k w i s e$ ). Otherwise Type $(H)=a$ (for anticlockwise). An example of edge type assignment is shown in Fig. 6.

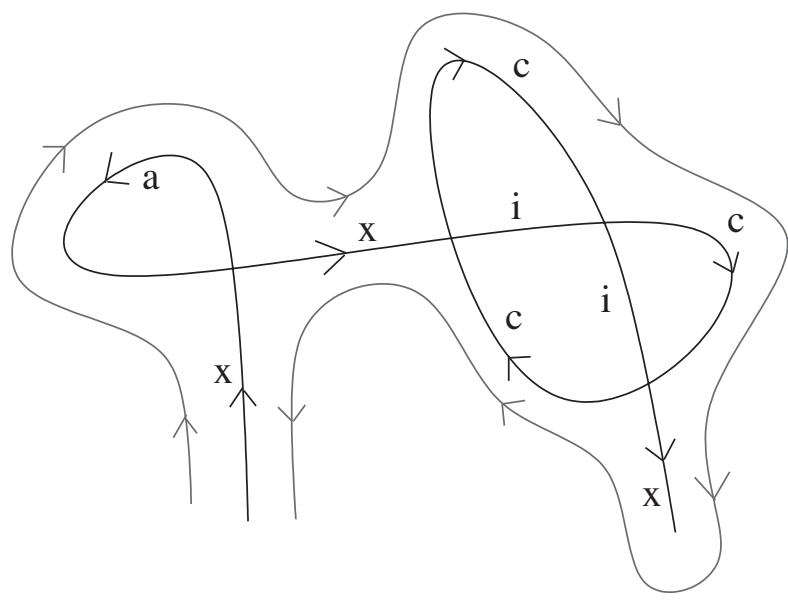

Fig. 6. Example of edge type assignment.

The algorithm for computing $\mathfrak{M}_{2}(P)$ from $P$ is as follows.

- Start with an empty string $A$;

- For each crossing node tip $T$ (processed in the standard order starting from $0 F$ ), do the following:

(1) If $T^{\prime}$ has already been processed, ignore $T$ and proceed to the next tip;

(2) Set $t:=\operatorname{Type}(H(T))$, and set $b:=\min \left\{T, T^{\prime}\right\}$ and $e:=\max \left\{T, T^{\prime}\right\}$ according to the ordering defined on the set of tips. The description of the edge is then 'bte';

(3) If $A$ is not empty, add a hyphen to $A$;

(4) Add the edge description to $A$.

Example 5.6. We have already seen that path (a) in Fig. 5 has $\mathfrak{M}_{1}$ path description J1-K0LA-J2-PLC-K1R. The $\mathfrak{M}_{2}$ path description is given by

$$
0 F i 1 B-0 L i 1 F-0 R i 2 B-S i O B-1 L i E-1 R i 2 R-2 F c 2 L .
$$

Proposition 5.7. $\mathfrak{M}_{2}$ is a well-defined path description method.

Proof 2. We must show that $\mathfrak{M}_{2}(P)=\mathfrak{M}_{2}(Q)$ implies $P \sim Q$. Let $P$ and $Q$ be paths for which $\mathfrak{M}_{2}(P)=\mathfrak{M}_{2}(Q)$. Now $P$ and $Q$ certainly have the same number of crossing points $N$. As described above, each tip can be given a unique label $k \alpha$, where $k$ is the node number and $\alpha \in\{F, L, R, B\}$. So there exists a map $f: P \rightarrow Q$, from tips of $P$ to tips of $Q$, such that every tip $T_{k \alpha}^{P}$ in $P$ is mapped to the tip $T_{k \alpha}^{Q}$ (i.e. the tip with the same label) in $Q$. Then for any edge $H_{P}$ in $P$ which joins $T_{k \alpha}^{P}$ to $T_{k^{\prime} \alpha^{\prime}}^{P}$ it follows that $f\left(T_{k \alpha}^{P}\right)=T_{k \alpha}^{Q}$ and $f\left(T_{k^{\prime} \alpha^{\prime}}^{P}\right)=T_{k^{\prime} \alpha^{\prime}}^{Q}$. Furthermore, since $\mathfrak{M}_{2}(P)=\mathfrak{M}_{2}(Q)$, we have that $T_{k \alpha}^{Q}$ and $T_{k^{\prime} \alpha^{\prime}}^{Q}$ are joined by an edge $S_{Q}$. So $f$ extends to a map which also sends edges of $P$ to edges of $Q$. This means that the two paths would be topologically equivalent if they were drawn on a sphere. As the two paths are drawn on a disk, they both have a set of external edges. As $\mathfrak{M}_{2}(P)=\mathfrak{M}_{2}(Q)$, not only are these external edges the same but they 
have the same orientation. This implies that the two paths are topologically equivalent which in turn implies that $P \sim Q$

\subsection{The conversion map}

The map $C(P): \mathfrak{M}_{1}(P) \rightarrow \mathfrak{M}_{2}(P)$ uses information from the tip table of $P$ to convert the path description $\mathfrak{M}_{1}(P)$ into the alternative path description $\mathfrak{M}_{2}(P)$. The basic structure of $C$ is given as follows.

First write out the complete path description, i.e. ' $J 0-\mathfrak{M}_{1}(P)-J E$ ', and label these instructions in order by $s_{0}, \ldots, s_{2 N}$. Then convert each instruction $s_{j}$ according the following map.

$$
\begin{aligned}
\operatorname{Px}([A C]) & \mapsto m F t_{j} m x \\
J n & \mapsto m y t_{j} n B \\
K n x([A C]) & \mapsto m y t_{j} n x .
\end{aligned}
$$

Here $m$ is the last number that appears before the current instruction (or $S$ if no numbers appear), $y$ is given by

- $F$, if $s_{j-1}=J m$ or $m=S$;

- $L$, if $s_{j-1} \in\{P R([A C]), K m R([A C])\}$;

- $R$, if $s_{j-1} \in\{P L([A C]), \operatorname{KmL}([A C])\}$,

and the $t_{j}=$ Type $\left(s_{j}\right)$ are the edge types to be determined. (Note that $S F$ is just interpreted as $S$.)

The algorithm for computing the edge types goes like this:

- Set $t_{j}:=i$ for all edges in the path.

- Traverse the outside of the path exactly once in a clockwise direction.

- For each edge $s_{j}$ travelled along:

(1) If $t_{j}=i$ then

- if the direction of $s_{j}$ matches the direction of traversal, set $t_{j}:=c$;

- otherwise set $t_{j}:=a$.

- If $t_{j} \neq i$ then set $t_{j}:=x$ (because we've already travelled along the edge once before).

In order to perform a traversal of the outer edges we need to find a node in the external region from which to start. We can then use the information from the tip table to follow round the outside edges in a clockwise manner. Finding this starting point is done as follows:

The algorithm remembers the last edge that was added in region 0 . This edge is guaranteed to border region 0 . If this edge is a join to $E$ then $E$ is in region 0 and we can start tracing from there. If not, the edge will have a direction (either $C$ or $A$ ). If the direction is $C$ we start from the edge's start tip. If the direction is $A$ we start from the edge's end tip.

So far we have a list of $\mathfrak{M}_{2}$ edge descriptions. The path description $\mathfrak{M}_{2}(P)$ is obtained by reversing any edge descriptions if necessary (so that they are all of the form $b t_{j} e$, with $b<e$ ), and then writing these descriptions in the appropriate order.

\subsection{Mirrors and reversals via $\mathfrak{M}_{2}$}

The $\mathfrak{M}_{2}$ path description of a mirror is obtained from the $\mathfrak{M}_{2}$ path description of the initial path by making the substitutions

$$
\begin{aligned}
& L \rightarrow R \\
& R \rightarrow L \\
& a \rightarrow c \\
& c \rightarrow a,
\end{aligned}
$$

and then reordering the edge descriptions as necessary. (Note that since edges of the form $n L \operatorname{tnR}$ or $n R t n L$ are not possible, each individual edge description of a mirrored edge will always be ordered correctly.)

Constructing $\mathfrak{M}_{2}$ path descriptions of reversals takes a bit more effort. First, it is clear that a path reversal will reorder the labels $S, v_{0}, v_{1}, \ldots, v_{N-1}, E$ of the nodes, but this reordering is not immediately obvious. We can obtain the new vertex ordering via the following reordering of the $\mathfrak{M}_{2}$ path description.

Recall that $\mathfrak{M}_{2}(P)=s_{0}-\cdots-s_{2 N}$, where each edge description $s_{j}$ is of the form $b_{j} t_{j} e_{j}$. Then there is a unique reordering $s_{0}^{\prime}-\cdots-s_{2 N}^{\prime}$ of the $\mathfrak{M}_{2}$ edge descriptions (with edge reversals $b t e \rightarrow$ etb performed if necessary) so that

- for each edge description $s_{j}^{\prime}=b_{j}^{\prime} t_{j} e_{j}^{\prime}$, the tips $e_{j}^{\prime}$ and $b_{j+1}^{\prime}$ are on the same node and opposite to each other;

- $b_{0}^{\prime}=E$ and $e_{2 N}^{\prime}=S$.

We denote this reordered description of $P$ by $\mathfrak{M}_{2}^{\prime}(P)$. Now, for $k=0, \ldots, N-1$, set $a_{k}$ to be the $(k+1)$ st distinct number appearing in $\mathfrak{M}_{2}^{\prime}(P)$ as we read through from left to right. Then the reordering of the nodes is given by 


$$
\begin{aligned}
S & \rightarrow E \\
v_{a_{k}} & \rightarrow v_{k} \\
E & \rightarrow S,
\end{aligned}
$$

and we define $\mathfrak{M}_{2}^{\prime \prime}(P)$ to be result of applying these substitutions to $\mathfrak{M}_{2}^{\prime}(P)$.

Now write $E=v_{N}$. Then for each $k \in\{S, 0,1, \ldots, N-1\}$, let $s_{k}^{\prime \prime}$ be the first edge description in $\mathfrak{M}_{2}^{\prime \prime}(P)$ of the form $m V t(k+1) W_{k+1}$, where $m \in\{S, 0,1, \ldots, k\}$ and $V$ is the tip label for $v_{m}$ (or absent if $m=S$ ), and $W_{k+1}$ is the tip label for $v_{k+1}$. This is the first edge appearing in the path description which connects to $v_{k+1}$. Since these edge descriptions will eventually represent joins (in the $\mathfrak{M}_{1}$ sense) from one node in the reversal $-P$ to a new node, it follows that every $W_{k+1}$ should be substituted by $B$. It is also clear that the existence of such an edge for each $k$ is guaranteed because all nodes $v_{k}$ must be eventually joined. Since the cyclic order of tip labels at any crossing node vertex is fixed (i.e. $F, R, B$, $L$, if we go round clockwise), the subtitutions for all tips are uniquely determined by the substitution $W_{k+1} \rightarrow B$. For example, if $W_{k+1}=L$ then we perform the permutation $(F, R, B, L) \rightarrow(L, F, R, B)$ at that node.

The last set of substitutions concern the edge types. Since a path reversal sends internal edges to internal edges and reverses the orientations on each edge, it follows that the required type substitutions are $a \rightarrow c$ and $c \rightarrow a$.

The path description $\mathfrak{M}_{2}(-P)$ of the reversal of $P$ is now obtained by applying the tip label and type substitutions to $\mathfrak{M}_{2}^{\prime \prime}(P)$, and then reordering as appropriate.

Example 5.8. Consider the path $P$ given by

$$
\mathfrak{M}_{2}(P)=0 F i 1 B-0 L i 1 F-0 R i 2 B-S i O B-1 L i E-1 R i 2 R-2 F c 2 L
$$

(i.e. path (a) in Fig. 5). The new edge descriptions for the mirror $P^{*}$ (see Fig. 5(b)) are computed as shown below:

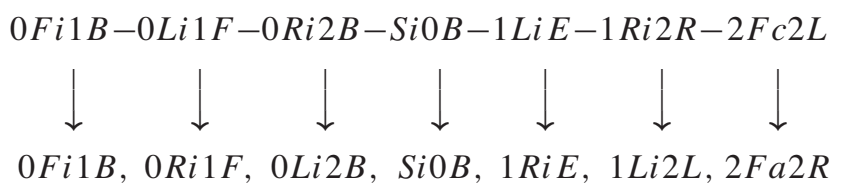

Reordering this gives us the $\mathfrak{M}_{2}$ path description of the mirror:

$$
\mathfrak{M}_{2}\left(P^{*}\right)=0 F i 1 B-0 L i 2 B-0 R i 1 F-S i 0 B-1 L i 2 L-1 R i E-2 F a 2 R .
$$

The construction of the $\mathfrak{M}_{2}$ path description of the reversal $-P$ (see Fig. 5(c)) runs as follows.

$$
\mathfrak{M}_{2}^{\prime}(P)=E i 1 L-1 R i 2 R-2 L c 2 F-2 B i 0 R-0 L i 1 F-1 B i 0 F-0 B i S .
$$

So the new ordering of the nodes is given by

$$
\begin{aligned}
& S \rightarrow E \\
& 0 \rightarrow 2 \\
& 1 \rightarrow 0 \\
& 2 \rightarrow 1 \\
& E \rightarrow S .
\end{aligned}
$$

Hence

$$
\mathfrak{M}_{2}^{\prime \prime}(P)=S i 0 L-0 R i 1 R-1 L c 1 F-1 B i 2 R-2 L i 0 F-0 B i 2 F-2 B i E .
$$

Now the joins on this path will be represented by the edges $\operatorname{SiOL}, 0 R i 1 R, 1 B i 2 R$ and $2 B i E$, so we require the following tip permutations:

$$
\begin{aligned}
& (0 F, 0 R, 0 B, 0 L) \rightarrow(0 L, 0 F, 0 R, 0 B) \\
& (1 F, 1 R, 1 B, 1 L) \rightarrow(1 R, 1 B, 1 L, 1 F) \\
& (2 F, 2 R, 2 B, 2 L) \rightarrow(2 R, 2 B, 2 L, 2 F) .
\end{aligned}
$$

After performing these substitutions, together with $a \rightarrow c$ and $c \rightarrow a$, we obtain

$$
\text { SiOB }-0 F i 1 B-1 F a 1 R-1 \text { Li2B }-2 F i 0 L-0 R i 2 R-2 L i E \text {. }
$$

The $\mathfrak{M}_{2}$ path description of the reversal of $P$ is then obtained by reordering this description in the appropriate way, i.e.

$$
\mathfrak{M}_{2}(-P)=0 F i 1 B-0 L i 2 F-0 R i 2 R-S i O B-1 F a 1 R-1 L i 2 B-2 L i E .
$$


The reader is invited to confirm that the path description of the mirror reversal of $P$ (see Fig. 5(d)) is given by

$$
\mathfrak{M}_{2}\left(-P^{*}\right)=0 F i 1 B-0 L i 2 L-0 R i 2 F-S i 0 B-1 F c 1 L-1 R i 2 B-2 R i E .
$$

(Try to do this verification using the three available methods, i.e. construct $\mathfrak{M}_{2}\left((-P)^{*}\right), \mathfrak{M}_{2}\left(-\left(P^{*}\right)\right)$ and $C\left(\mathfrak{M}_{1}\left(-P^{*}\right)\right.$ ) from the path descriptions already constructed.)

\section{The Immint Package}

The computer implementation of the algorithm is split into two modules. The first module immint (written in C) is the path generator.

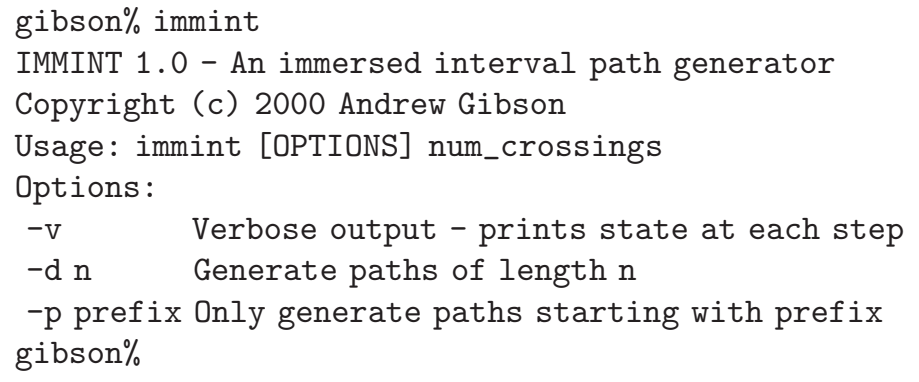

It generates $\mathfrak{M}_{1}$ path descriptions for all valid paths $P$ with a given number of double points $N$. Then, for each of these paths, it computes the $\mathfrak{M}_{2}$ path descriptions of $P, P^{*},-P$ and $-P^{*}$. For example, immint produces the following for $N=1$.

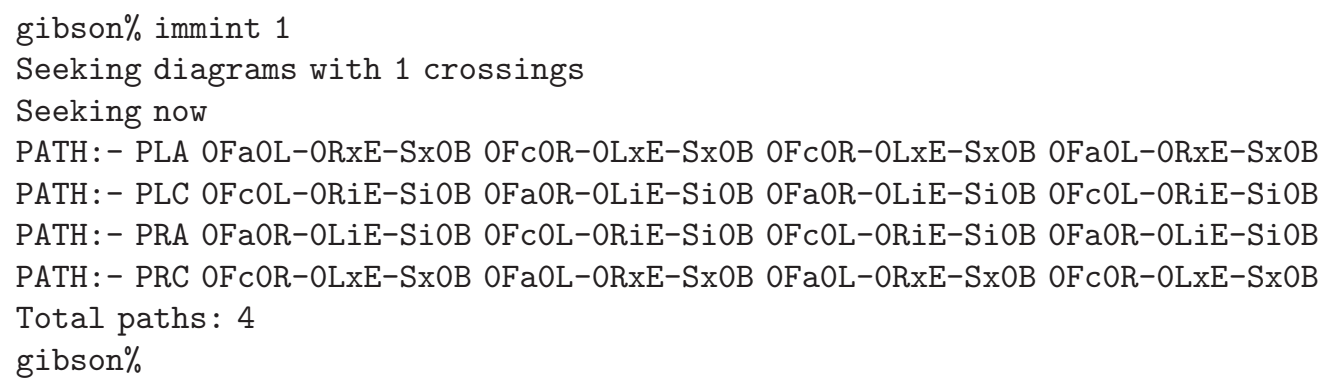

The output of the immint module is then piped to the second module immsum. This second module is a perl script which carries out the elimination part of the algorithm. The final output in list format (using the -1 flag) is given below for $N=1$.

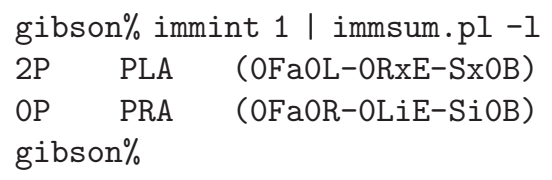

In this listing, entries in the first column are of the form $\mathrm{nP}$, where $\mathrm{n}$ gives the number of external end points, and $\mathrm{P}$ indicates that the free divide is prime. In particular, if $\mathrm{n}=2$ then the free divide is also a divide.

As a slightly more interesting example, immint generates a list of 30 paths with $N=2$. The result after piping this list to immsum is as follows (where a $\mathrm{C}$ in the first column indicates a compound free divide).

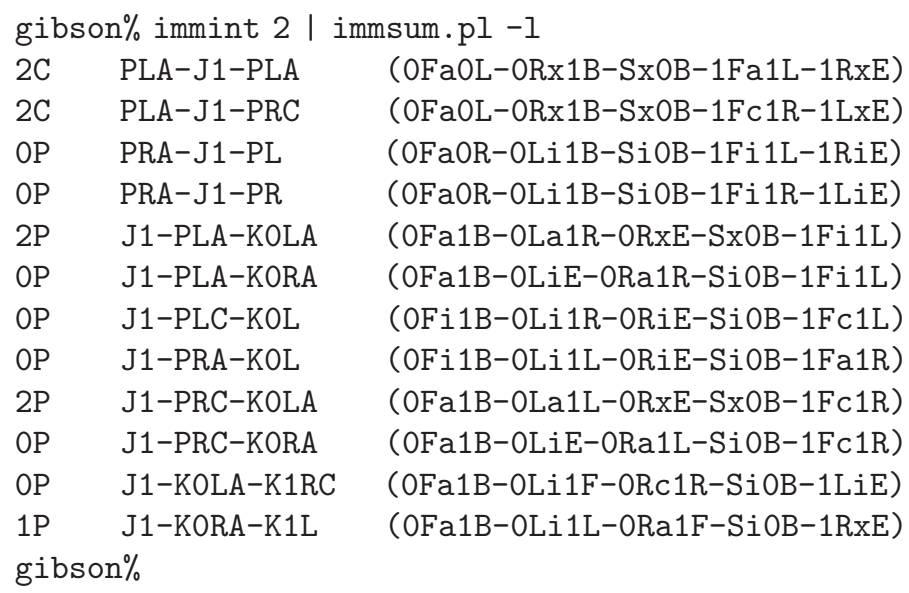




\section{Free Divide Totals}

The immsum script can also produce a tabulated summary of how many free divides there are for a given number of crossing points. This is done by using the $-\mathrm{t}$ flag. In this section we reproduce the output for the command immint $N$ I immsum.pl -t, for $N \leq 8$.

In addition to free divide totals, each table also indicates how long the machine took to complete the task. The method used to measure this elapsed time is very crude. It is calculated by taking a timestamp $t_{\text {begin }}$ in seconds as soon as the immsum script commences, and subtracting it from a timestamp $t_{\text {end }}$ taken when the script finishes. Therefore an elapsed time of 0 actually means it took less than a second. This data was obtained on a Pentium III machine running under Linux.

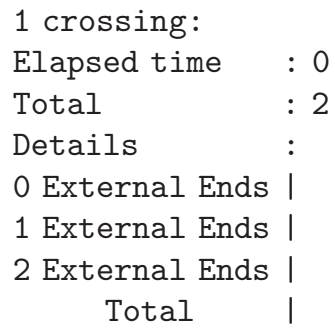

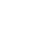

.

3 crossings:

Elapsed time :0

Total : 79

Details :

1 External Ends |

2 External Ends | Total

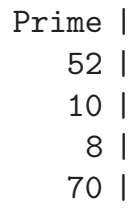

\begin{tabular}{|c|}
\hline Prime | \\
\hline 475 । \\
\hline 91 | \\
\hline 381 \\
\hline 60 \\
\hline
\end{tabular}

5 crossings:

Elapsed time : 8

Total

Details

0 External Ends |

1 External Ends |

2 External Ends | Total

\begin{tabular}{r|r} 
Compound & Total \\
0 & 1 \\
0 & 0 \\
0 & 1 \\
0 & 2
\end{tabular}

\begin{tabular}{r|r} 
Compound & Total \\
0 & 7 \\
0 & 1 \\
2 & 4 \\
2 & 12
\end{tabular}

\begin{tabular}{r|r} 
Compound & Total \\
0 & 52 \\
2 & 12 \\
7 & 15 \\
9 & 79
\end{tabular}

\begin{tabular}{r|r} 
Compound & Total \\
2 & \\
28 & 477 \\
44 & 119 \\
74 & 82 \\
& 678
\end{tabular}

\begin{tabular}{r|r} 
Compound & Total \\
23 & 4653 \\
304 & 1138 \\
273 & 496 \\
600 & 6287
\end{tabular}

6 crossings:

Elapsed time : 93

Total

: 62232 


\begin{tabular}{|c|c|c|c|}
\hline Details & Prime | & Compound I & Total \\
\hline 0 External Ends & 47492 | & $344 \mid$ & 47836 \\
\hline 1 External Ends & 7798 | & 3076 । & 10874 \\
\hline 2 External Ends & $1534 \mid$ & $1988 \mid$ & 3522 \\
\hline Total & $56824 \mid$ & 5408 | & 62232 \\
\hline \multicolumn{4}{|l|}{7 crossings: } \\
\hline Elapsed time & \multicolumn{3}{|l|}{ : 1200} \\
\hline Total & \multicolumn{3}{|l|}{ : 633172} \\
\hline Details & Prime | & Compound I & Total \\
\hline 0 External Ends & $497126 \mid$ & 4273 । & 501399 \\
\hline 1 External Ends & 74337 | & 30486 I & 104823 \\
\hline 2 External Ends & 11711 & 15239 & 26950 \\
\hline Total & $583174 \mid$ & 49998 I & 633172 \\
\hline \multicolumn{4}{|l|}{8 crossings: } \\
\hline Elapsed time & \multicolumn{3}{|l|}{ : 12474} \\
\hline Total & \multicolumn{3}{|l|}{ : 6566426} \\
\hline Details & Prime | & Compound I & Total \\
\hline 0 External Ends & 5274128 | & 51054 & 5325182 \\
\hline 1 External Ends & 720549 & 301102 | & 1021651 \\
\hline 2 External Ends & 95971 | & 123622 & 219593 \\
\hline Total & 6090648 I & 475778 | & 6566426 \\
\hline
\end{tabular}

A summary of the total number of free divides up to 8 double points is shown in Table 4 together with the elapsed time data. As a measure of algorithm efficiency, the elapsed time $t_{\text {end }}-t_{\text {begin }}$ becomes increasingly approximate as $N$ increases. This is because it doesn't take into account the fact that the processor is busy with other jobs. However, we note that for $N \geq 4$ the elapsed time increases (very) approximately by a factor of 10 on each increment of $N$. Thus we estimate it would take just over a day to compute the total for $N=9$ on the same machine.

Table 4. Summary of total numbers of free divides and elapsed time for $N \leq 8$.

\begin{tabular}{|c|c|c|}
\hline$\sharp$ double points & elapsed time $(\mathrm{s})$ & $\sharp$ free divides \\
\hline 1 & 0 & 2 \\
2 & 0 & 12 \\
3 & 0 & 79 \\
4 & 1 & 678 \\
5 & 8 & 6287 \\
6 & 93 & 62232 \\
7 & 1200 & 633172 \\
8 & 12474 & 6566426 \\
\hline
\end{tabular}

\section{Free Divide Tabulation using a Distributed Computer System}

Once we get above a certain number of double points (about 9) it becomes less practical to run the whole path listing process on a single machine. This is for two reasons:

(1) The time it takes a machine to generate all paths gets increasingly longer with each increment of double points.

(2) If we wish to generate and store the full list of paths then this can take up an increasingly large amount of disk space with each increment of double points.

To address the first problem, we describe how the Immint package can be extended to perform parallel batch processing using a distributed computer system.

The immint module allows the user to generate a list of valid path prefixes of a given length. For example, the command 'immint -d 5 10' will produce a list of valid path prefixes for 10 crossing points, each prefix containing 5 instructions, i.e.

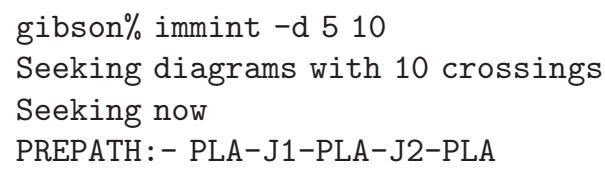


divides and oriented divides. The data returned by this script can then be fed into the knotfind module of the Knotscape software (albeit after a few modifications), and the knots can be looked up. Knotscape, written by Hoste, Thistlethwaite et al, contains a library of all prime knots up to 16 crossings.

Note that this scheme can be expanded to incorporate the parallel batch processing discussed in the previous section. In this case each slave machine can use pdivide and knotfind to look up the links of the paths in their respective batches. Collation then takes place after the knot types have been determined, i.e. the output will be a list of knots with known unknotting number.

Remark 5. For this scheme to work well for high $N$, Knotscape's knotfind module needs to be expanded so that it can cope with Dowker-Thistlethwaite codes of length greater than 50. In addition, the reduction algorithm used in the pdivide script could be optimised further.

\section{REFERENCES}

[1] A’Campo, N., "Le groupe de monodromie du déploiement des singularité isolées de courbes planes I," Math. Ann., 213: 1-32 (1975).

[2] A'Campo, N., "Le groupe de monodromie du déploiement des singularité isolées de courbes planes II," Actes du Congrès International des Mathmaticiens, Vancouver, 395-404 (1974).

[3] A'Campo, N., "Generic immersions of curves, knots, monodromy and gordian numbers," Publ. Math. de l'H.E.S., 88: 151169 (1998).

[4] Gibson, W., "Links and Gordian numbers associated with generic immersions of trees," preprint, appears in Proc. 'Art of Low Dimensional Topology 7', Kyoto (2001).

[5] Gibson, W., "Oriented divides and plane curve braids," J. Knot Theory and its Ramifications, 11: $973-1016$ (2002).

[6] Gibson, W., and Ishikawa, M., "Links of oriented divides and fibrations in link exteriors," Osaka J. Math., 39: 681-703 (2002).

[7] Gibson, W., and Ishikawa, M., "Links and Gordian numbers associated with generic immersions of intervals," Topology Appl., 123: 609-636 (2002).

[8] Gusein-Zade, S. M., "Intersection matrices for certain singularities of functions of two variables," Funct. Annal. Appl., 8: 1013 (1974).

[9] Gusein-Zade, S. M., "Dynkin diagrams of singularities of functions of two variables," Funct. Annal. Appl., 8: $295-300$ (1974).

[10] Gusein-Zade, S. M., "The monodromy groups of isolated singularities of hypersurfaces," Russ. Math. Surv., 32: 23-69 (1977).

[11] Ishikawa, M., "Half plane models for divides, their knots and Dowker-Thistlethwaite codes," Interdisciplinary Inf. Sci., 7: 17$24(2001)$. 